\title{
Comparison of BacT/ALERT 3D, Lowenstein-Jensen medium and Middlebrook 7H10/7H11 biplate for recovering mycobacteria from clinical specimens
}

Published online: 29 June 2005

(C) Springer-Verlag 2005

Rapid and sensitive detection of mycobacteria is essential for the successful treatment and control of mycobacterial infections. It is considered good laboratory practice to combine a liquid medium with at least one solid medium for culturing mycobacteria from clinical specimens. Automated systems with liquid media provide faster results and are more sensitive, while solid media help to detect mixed infections and can detect mycobacteria that do not grow in the broth medium. In order to evaluate the BacT/ALERT 3D system (bioMérieux, Durham, NC, USA) and to find the optimal combination of media, we compared the results achieved with BacT/ALERT 3D, Lowenstein-Jensen (L-J) medium (Bio-Rad, Marne-la Coquette, France), and the Middlebrook 7H10/7H11 biplate (Becton Dickinson, Sparks, MD, USA) on consecutive clinical specimens collected within a period of 26 months.

A total of 6,250 clinical samples (4,051 respiratory specimens, 1,209 normally sterile body fluids, 292 biopsy specimens, 247 blood specimens, 344 urine specimens and 304 other specimens) from 3,247 patients were tested for the presence of mycobacteria. Specimens with a different panel of media (blood and urine) and specimens contaminated with bacteria other than mycobacteria were excluded from the evaluation.

Tissue specimens were crushed and homogenized. Homogenized tissue, respiratory specimens and other specimens from contaminated sites were decontaminated with sodium dodecyl sulfate- $\mathrm{NaOH}$ as described by Tacquet and Tisson [1]. Liquids from sterile sites were concentrated by centrifugation $(3000 \mathrm{~g}, 15 \mathrm{~min})$. Aliquots of $0.3-0.5 \mathrm{ml}$ were inoculated onto a L-J slant, a Middlebrook 7H10/7H11 selective agar biplate with carbenicillin, polymyxin $\mathrm{B}$, trimethoprim lactate, and amphotericin $\mathrm{B}$, and into a BacT/ALERT MP bottle. For specimens from

H. Adler $(\bowtie) \cdot$ C. Straub $\cdot$ R. Frei

Microbiology Laboratory, University Hospital Basel,

Petersgraben 4

4031 Basel, Switzerland

e-mail: hadler@uhbs.ch

Tel.: +41-61-2654248

Fax: +41-61-2655355 contaminated sites, $0.5 \mathrm{ml}$ of MB/BacT Antibiotic Supplement (bioMérieux) was added to the BacT/ALERT MP bottle.

The incubation time was 8 weeks. L-J slants were incubated at $36^{\circ} \mathrm{C}$ in ambient air and 7H10/7H11 biplates were incubated at $36^{\circ} \mathrm{C}$ in $5 \% \mathrm{CO}_{2}$. Both media were examined visually once a week for the appearance of colonies. The BacT/ALERT MP bottles were handled according to the manufacturer's instructions. Detection of mycobacterial growth in the BacT/ALERT 3D system is based on the colorimetric detection of $\mathrm{CO}_{2}$, and the cultures are continuously monitored by the automated system.

Ziehl-Neelsen staining was used to confirm the presence of acid-fast bacilli in all positive media. Mycobacterium tuberculosis was identified using the Cobas Amplicor MTB system (Roche Diagnostics, Basel, Switzerland) combined with the following biochemical tests: production of niacin and heat-stable catalase and reduction of nitrate. Nontuberculous mycobacteria (NTM) were identified using nucleic acid probes (Gen-Probe, San Diego, CA, USA) or by $16 \mathrm{~S}$ rDNA sequencing [2] with the MicroSeq 500 16S rDNA Bacterial Sequencing Kit (Applied Biosystems, Foster City, CA, USA). Sequences were compared with those available in RIDOM, a comprehensive 16S rRNA sequence database for mycobacteria [3].

A total of 345 isolates of mycobacteria were evaluated. Seventeen specimens were contaminated with organisms other than mycobacteria and consequently excluded; two were in BacT/ALERT MP bottles, one was on L-J medium and 14 were on Middlebrook 7H10/7H11 biplates.

Mycobacterium tuberculosis was identified in 285 specimens collected from 100 patients. Sixty isolates of NTM were recovered from 58 clinical specimens of 33 patients. The NTM isolates comprised the following species: $M$. avium/intracellulare (26), $M$. chelonae (1), M. flavescens (1), M. fortuitum (1), M. gordonae (7), M. kansasii (5), M. lentiflavum/simiae (1), M. malmoense (1), M. marinum (2), M. peregrinum (1), M. phlei (1), $M$. septicum/peregrinum (3), $M$. terrae complex (1), M. xenopi (7), unidentified Mycobacterium spp. (2). Mixed cultures with Mycobacterium avium and Mycobacterium 
Table 1 Results achieved using different media to detect mycobacteria

\begin{tabular}{llllllll}
\hline Mycobacteria & BacT/ALERT & L-J & 7H10/7H11 & $\begin{array}{l}\text { BacT/ALERT } \\
\text { plus L-J }\end{array}$ & $\begin{array}{l}\text { BacT/ALERT } \\
\text { plus 7H10/7H11 }\end{array}$ & $\begin{array}{l}\text { L-J plus } \\
7 H 10 / 7 H 11\end{array}$ \\
\hline M. tuberculosis & & & & & & \\
\hline Smear positive & $158(95 \%)$ & $159(96 \%)$ & $153(92 \%)$ & $165(99 \%)$ & $163(98 \%)$ & $165(99 \%)$ \\
Smear negative & $104(87 \%)$ & $97(82 \%)$ & $84(71 \%)$ & $116(97 \%)$ & $111(93 \%)$ & $107(90 \%)$ & 119 \\
Total $^{\text {a }}$ & $262(92 \%)$ & $256(90 \%)$ & $237(83 \%)$ & $281(99 \%)$ & $274(96 \%)$ & $272(95 \%)$ & 285 \\
NTM $^{\text {b }}$ & $48(80 \%)$ & $21(35 \%)$ & $38(63 \%)$ & $55(91 \%)$ & $55(91 \%)$ & $45(75 \%)$ & 60 \\
\hline
\end{tabular}

L-J, Lowenstein-Jensen; NTM, non-tuberculous mycobacteria

${ }^{a}$ Significant differences $(p<0.05)$ : BacT/ALERT vs 7H10/7H11, L-J vs 7H10/7H11, BacT/ALERT vs BacT/ALERT plus L-J, BacT/ALERT vs BacT/ALERT plus 7H10/7H11, L-J vs BacT/ALERT plus L-J, L-J vs BacT/ALERT plus 7H10/7H11, L-J vs L-J plus 7H10/7H11, 7H10/7H11 vs BacT/ALERT plus L-J, 7H10/7H11 vs BacT/ALERT plus 7H10/7H11, 7H10/7H11 vs L-J plus 7H10/7H11, BacT/ALERT plus L-J vs L-J plus 7H10/7H11

${ }^{\mathrm{b}}$ Significant differences $(p<0.05)$ : BacT/ALERT vs L-J, BacT/ALERT vs 7H10/7H11, L-J vs 7H10/7H11, L-J vs BacT/ALERT plus L-J, L-J vs BacT/ALERT plus 7H10/7H11, L-J vs L-J plus 7H10/7H11, 7H10/7H11 vs BacT/ALERT plus L-J, 7H10/7H11 vs BacT/ALERT plus 7H10/7H11, BacT/ALERT plus L-J vs L-J plus 7H10/7H11, BacT/ALERT plus 7H10/7H11 vs L-J plus 7H10/7H11

kansasii were detected twice. Isolation rates achieved with each medium are given in Table 1.

The BacT/ALERT 3D system is a fully automated system based on the detection of $\mathrm{CO}_{2}$ produced by the proliferating mycobacteria. The main differences between the BacT/ALERT 3D model and its predecessor, the $\mathrm{MB} / \mathrm{BacT}$, are in the system's electronic and data management components. Only a few studies have evaluated the new BacT/ALERT 3D system, and they assessed the BacT/ALERT 3D system in comparison with either L-J medium alone [4-6] or L-J medium and the Bactec 460 TB System [7]. In those studies, the recovery rates reported for the BacT/ALERT 3D system ranged from 85 to $100 \%$ for M. tuberculosis and 52 to $100 \%$ for NTM. However, the two studies that reported recovery rates of $100 \%$ for NTM included only five and eight isolates of NTM, respectively $[4,5]$. In comparison, our study included a large number of isolates, and we found recovery rates of $92 \%$ for $M$. $t u$ berculosis and $80 \%$ for NTM using the BacT/ALERT 3D system.

In summary, the BacT/ALERT 3D system proved to be a highly sensitive culture system when compared to solid media. There were no significant differences in the recovery rates achieved using BacT/ALERT 3D alone and the combination of the two solid media, but the recovery rates of BacT/ALERT 3D were enhanced for both $M$. tuberculosis and NTM when the system was used in combination with a solid medium. The combination of BacT/ALERT 3D plus a solid medium offers the additional advantage of facilitating detection in cases of contamination of one medium or in cases of mixed mycobacterial cultures, which are more readily detected on solid media.

\section{References}

1. Tacquet A, Tisson F (1961) Résultat de l'isolement des mycobactéries par le lauryl-sulfate de sodium. Ann Inst Pasteur 100:676-680

2. Rogall T, Flohr T, Bottger EC (1990) Differentiation of $M y$ cobacterium species by direct sequencing of amplified DNA. J Gen Microbiol 136:1915-1920

3. Harmsen D, Rothganger J, Frosch M, Albert J (2002) RIDOM: Ribosomal differentiation of medical micro-organisms database. Nucleic Acids Res 30:416-417

4. Carricajo A, Fonsale N, Vautrin AC, Aubert G (2001) Evaluation of BacT/Alert 3D liquid culture system for recovery of mycobacteria from clinical specimens using sodium dodecyl (lauryl) sulfate- $\mathrm{NaOH}$ decontamination. J Clin Microbiol 39:3799-3800

5. Alcaide F, Gali N, Dominguez J, Berlanga P, Blanco S, Orus P, Martin R (2003) Usefulness of a new mycobacteriophage-based technique for rapid diagnosis of pulmonary tuberculosis. J Clin Microbiol 41:2867-2871

6. Angeby KA, Werngren J, Toro JC, Hedstrom G, Petrini B, Hoffner SE (2003) Evaluation of the BacT/ALERT 3D system for recovery and drug susceptibility testing of Mycobacterium tuberculosis. Clin Microbiol Infect 9:1148-1152

7. Piersimoni C, Scarparo C, Callegaro A, Tosi CP, Nista D, Bornigia S, Scagnelli M, Rigon A, Ruggiero G, Goglio A (2001) Comparison of $\mathrm{MB} /$ Bact alert $3 \mathrm{D}$ system with radiometric BACTEC system and Lowenstein-Jensen medium for recovery and identification of mycobacteria from clinical specimens: a multicenter study. J Clin Microbiol 39:651-657 